\title{
MAGNETIC FIELDS: OBSERVATIONS AND TOPOLOGY
}

\author{
J. D. LANDSTREET \\ Department of Astronomy, University of Western Ontario, London, \\ Ontario, Canada N6A 3K7, and Observatoire Midi-Pyrénées, \\ 14 ave. Edouard Belin, 31400 Toulouse, France
}

\begin{abstract}
To understand the complex physical processes revealed by the wide variety of surface chemical abundances and the inhomogeneous distributions of various elements over the surfaces of magnetic Ap and Bp stars, it is important to be able to describe the surface magnetic field structure. For most magnetic stars, the available observations are far from sufficient to define the surface magnetic structure even with low resolution, but more extensive and constraining observations are gradually becoming available for a small set of stars. This paper discusses the extent to which the surface field structure can be defined for stars for which only the usual measurements (essentially the variation of the mean longitudinal field strength $B_{l}$ with rotational phase) are available, and how further information (variation of mean field modulus $B_{a}$ with phase, line profile observations, and circular and linear spectropolarimetry of absorption lines or broad-band radiation) can be used to obtain a much more detailed picture of the magnetic field structure.
\end{abstract}

\section{INTRODUCTION}

A large variety of physical processes occur in the atmospheres and envelopes of upper main sequence stars that have not yet been fully incorporated into stellar models, or even securely identified and understood. These processes include convection, turbulence, meridional circulation currents, diffusion of trace elements within the dominant hydrogen plasma, and mass loss through stellar winds. The most compelling observational evidence for the occurrence of most or all of these poorly understood processes in upper main sequences stars is the remarkable variety of surface chemical compositions observed in such stars, clearly in the obviously peculiar stars (Takada-Hidai 1991; Ryabchikova 1991; Dworetsky 1993; Cowley 1993), but also in more nearly normal stars such as Vega or Sirius (Cowley 1991; Adelman 1993).

The chemically peculiar stars play a key role in our efforts to understand the relevant physics, since it is in these stars that the effects of the various processes acting below, in and above the stellar atmospheres are most clearly visible. Among such stars, the magnetic chemically peculiar stars are particularly important. The magnetic stars show the most extreme chemical peculiarities of all, and some have strongly inhomogeneous distributions of 
certain chemical elements over their surfaces. From the observed facts that the strong spectrum variability that reveals chemical inhomogeneities is found only in magnetic peculiar stars (chemically peculiar but non-magnetic stars are not generally detectable spectrum variables), as well as the apparent association of extrema of spectral variation with an approach of the line of sight to one of the poles of the roughly dipolar field distribution, we deduce that the inhomogeneities are related to - probably even caused by - the magnetic field structure. It thus becomes important to understand the magnetic field geometry of a sample of representative magnetic stars in order to make progress in understanding the physics that underlies the chemical separation producing the chemical anomalies in these stars. This paper will review efforts to deduce from observations what the magnetic field structure of some such stars may be.

Several recent reviews have discussed related material. The general topic of magnetic fields in hot stars has been surveyed by Landstreet (1992a), while fields observed in upper and lower main sequence stars and in white dwarfs are discussed by Landstreet (1992b). The deduction of field strength and structure from spectral observations has been reviewed by Mathys (1989; 1993). Because of the existence of these reviews, the present paper does not need to be extremely detailed.

In this paper, we shall look first briefly at how constraints on stellar magnetic field structure may be derived from observations of spectral line profiles and (spectro-)polarimetry, using the physics of the Zeeman effect. The following section will survey simple inferences and modelling that may be done on the basis of the kinds of observations available for the majority of known magnetic upper main sequence stars. The final section will examine the improvement in modelling possibilities resulting when more extensive observational material is available, and will discuss the results of such modelling from the past few years.

\section{OBSERVATIONAL DATA}

We start by reviewing the basic physics underlying measurements of the magnetic field of a main sequence star, measurements that we would like to use to infer the distribution of the magnetic vector field over the surface of the star.

Field measurements for non-degenerate stars are based on the changes that occur in spectral lines when a free atom is immersed in a magnetic field, changes that are collectively referred to as the Zeeman effect. In the absence of a magnetic field, a particular atom has a series of discrete energy levels. When a field is imposed, each of these levels, of total angular momentum quantum number $\mathrm{J}$, splits in general into $2 \mathrm{~J}+1$ states (sometimes called magnetic substates), uniformly separated in energy by an amount which is proportional to the applied field $B$ (as long as the energy separation is small compared to the fine-structure splitting). As a result, each transition that produces a single spectral line in the spectrum when no field is present leads, in the presence of a field, to a group of closely spaced spectral lines: the line splits into a number of components. For atoms in LS coupling, these lines may be divided into three groups with slightly different properties: the $\pi$ components, produced by the transitions in which the magnetic quantum number $m$ changes by $\Delta m=0$, 
are symmetrically spread around the wavelength at which the transition occurs when no field is applied. Transitions for which $\Delta m=+1$ or -1 , the $\sigma$ components, have wavelengths above and below the zero-field wavelength, with transitions in which $\Delta m=+1$ on one side of the unsplit line and transitions in which $\Delta m=-1$ on the other. The wavelength separations of the individual line components, and between the centroids of the $\pi$ and the $\sigma$ groups, are proportional to the applied field $B$. Furthermore, the $\pi$ and $\sigma$ components have different polarization properties. In a field aligned parallel to the line of sight to the observer, the $\pi$ components vanish, and the two groups of $\sigma$ components have opposite circular polarizations. If the field is perpendicular to the line of sight, the $\pi$ components (in absorption) are linearly polarized normal to the field direction, and both $\sigma$ components are linearly polarized parallel to the field.

Both the line splitting and the polarization properties of the line components lead to practical and useful methods of measuring the magnetic field of an upper main sequence star. The splitting up of a single line into a number of components with wavelength separations that are proportional to the field strength leads at first to a widening of the line over its zero-field width, and then with increasing field to the line breaking up into distinct components (examine for example the detailed profiles shown by Landstreet 1988, Landstreet et al. 1989, or Mathys et al. 1993). Observation of this effect provides a measure of the field modulus $B$ averaged over the visible hemisphere (the mean field modulus $B_{s}$ ). Similarly, if the field has a relatively simple structure, the difference in circular polarization of the two $\sigma$ components may be detected in the line profile even after integration over the visible disk, as a difference in mean wavelength of the line as observed in right and in left circularly polarized light. This effect is proportional to the line of sight component of the field averaged over the visible hemisphere (the mean longitudinal field $B_{l}$ ). If the star has an appreciable projected rotational velocity, the effect of spreading out in the line profile of contributions from different slices on the star creates additional complexity in the line, but also offers additonal possibilities for deriving information about the field structure (Mathys 1993). Finally, in a strong field the trasverse component can produce observable linear polarization which can also be useful for field diagnosis (Landolfi et al. 1993; Leroy et al. 1993).

To get some more explicit idea of how these aspects of the line splitting and polarization appear in the spectrum of a star, it is illuminating to consider briefly and in a schematic way the effects of radiative transfer on a spectral line split by the Zeeman effect into a simple triplet (one $\pi$ and two $\sigma$ components). When a magnetic field is present, the usual equation of transfer becomes four equations describing the transfer of the four Stokes components. These may be written, in LTE and with the neglect of anomalous dispersion, as

$$
\begin{aligned}
& \mu d I / d \tau=\left(1+\eta_{I}\right) I+\eta_{Q} Q+\eta_{U} U-\left(1+\eta_{I}\right) B_{\nu} \\
& \mu d Q / d \tau=\eta_{Q} I+\left(1+\eta_{I}\right) Q-\eta_{Q} B_{\nu} \\
& \mu d U / d \tau=\eta_{U} I+\left(1+\eta_{I}\right) U-\eta_{U} B_{\nu} \\
& \mu d V / d \tau=\eta_{V} I+\left(1+\eta_{I}\right) V-\eta_{V} B_{\nu}
\end{aligned}
$$

where $I=I(\mu, \lambda), Q, U$ and $V$ are the Stokes components, $\tau$ is the optical depth in the continuum, $\mu=\cos \theta, B_{\nu}$ is the Planck function and the various $\eta$ 's 
are appropriate line-to-continuum opacity ratios that are linear combinations of the polarized line-to-continuum ratios for the $\pi$ and $\sigma$ components of the Zeeman-split line, defined precisely by Rees (1987) or Mathys (1989).

If we assume that all the $\eta$ 's are independent of depth, and that $B_{\nu}$ varies as a function of continuum optical depth as $B_{\nu}=B_{0}(1+\beta \tau)$, where $\beta$ is a constant, these four equations form a system of four linear, first-order differential equations with constant coefficients and specified driving terms (this is clearly the analogue of the Milne-Eddington approximation for the normal transfer equation). In this case, an analytic solution exists for the emergent Stokes components:

$$
\begin{aligned}
& I(\mu)=B_{0}\left[1+\beta \mu\left(1+\eta_{I}\right) / \Delta\right] \\
& Q(\mu)=-B_{0} \mu \beta \eta_{Q} / \Delta \\
& U(\mu)=-B_{0} \mu \beta \eta_{U} / \Delta \\
& V(\mu)=-B_{0} \mu \beta \eta_{V} / \Delta
\end{aligned}
$$

where $\Delta=\left(1+\eta_{I}^{2}\right)-\eta_{Q}^{2}-\eta_{U}^{2}-\eta_{V}^{2}$. In the particular case of a Zeeman triplet, for a field small enough that the magnetic line splitting $\Delta \lambda_{z}$ is less than the thermal width $\Delta \lambda_{D}$ of the line (typically valid as long as the local field satisfies $B \leqslant 1$ $\mathrm{kG}$ ) these solutions become, to first order in the ratio $\Delta \lambda_{Z} / \Delta \lambda_{D}$,

$$
\begin{aligned}
& I(\mu, \lambda)=B_{0}+B_{0} \mu \beta /[1+\eta(\lambda)] \\
& Q(\mu, \lambda)=0 \\
& U(\mu, \lambda)=0 \\
& V(\mu, \lambda)=-\bar{g} \Delta \lambda_{z} B_{z}[d I(\mu, \lambda) / d \lambda),
\end{aligned}
$$

where $\Delta \lambda_{z}=e \lambda^{2} / 4 \pi m c^{2}$ is the Lorentz unit, $\bar{g}$ (the effective Landé factor) is a number of order unity that varies from one transition to another, and $\eta(\lambda)$ is simply the intrinsic line-to-continuum opacity ratio in the absence of a magnetic field.

Now we must integrate this solution over the visible hemisphere of the observed star. This can be done analytically if we assume that the star is nonrotating. In this case, the line profile is given by

$$
\bar{I}(\lambda)=(1 / \pi) \int_{0}^{2 \pi} d \phi \int_{0}^{\pi / 2} \sin \theta \cos \theta I(\mu, \lambda) d \theta=B_{0}+(2 / 3) B_{0} \beta /[1+\eta(\lambda)] .
$$

Because the local solution does not contain any reference to the magnetic field (the $\eta(\lambda)$ that appears in $I(\mu, \lambda)$ is the profile of the non-magnetic line), the integrated profile is also (to first order) independent of the field. Similarly, the integrated values of $Q$ and $U$, which are locally everywhere zero, vanish. The line is not linearly polarized. The only information about the field is contained in the $V$ Stokes component, which has the simple form

$$
\bar{V}(\lambda)=\bar{g} \Delta \lambda_{z} B_{l}[d \bar{I}(\lambda) / d \lambda]
$$

where

$$
B_{l}=(3 \pi / 2) \int_{0}^{2 \pi} d \phi \int_{0}^{\pi / 2} \sin \theta \cos ^{2} \theta B_{z} d \theta
$$

is the mean longitudinal field, a weighted average of the line-of-sight 
component $B_{z}$ of the field over the visible hemisphere. That is, a weak magnetic field is detectable (to first order) only if it has a non-zero mean longitudinal field (which will only be true if the field structure is not very complex). And even if the field is detectable, the only information one has about its structure is the value of a single moment of $B_{z}$ (which of course may be measured a number of times from different directions as the star turns). Although we have derived this result under a number of restrictive approximations, it is generally true that a single moment of the line-of-sight component is all that can reasonably be measured for stars with local fields of less than, say, $2 \mathrm{kG}$. Since perhaps half of the known magnetic Ap stars have fields of this size, the information about the fields of many magnetic stars is in fact severely limited.

For field strengths large enough to significantly distort the local line profile (i.e. $B \geq 2 \mathrm{kG}$ ), all the Stokes components are sensitive to the field, and considerably more information about the field structure is accessible to high-resolution spectroscopy and spectropolarimetry. For slowly rotating stars with sufficiently large fields $(B \geq 4 \mathrm{kG})$, line splitting is directly visible in favourable lines. In this case, since the line splitting is proportional to $B$, another simple field moment, the mean field modulus $B_{d}$, may be obtained from high-resolution spectra (Mathys 1990; Mathys and Lanz 1991). Modest rotation (say, $20 \leqslant v \sin i \leqslant 50 \mathrm{~km} \mathrm{~s}^{-1}$ ) makes still more information accessible, by separating in wavelength contributions from different parts of the star via the Doppler effect, but this information requires more effort to interpret.

Thus for stars with weak fields, the only information that is available is the variation of the weighted mean of the line-of-sight field component as the star rotates. Only for stars with relatively large fields can line splitting, or more complex circular (or linear) polarization in spectral lines be measured. Thus it is only the large-field stars for which there is at present much hope of obtaining detailed maps of the magnetic field geometry.

It must be emphasized that even with high resolution observations at a number of rotational phases, a map of the surface will have relatively modest surface resolution. For example, if we have measurements of a single line profile $\bar{I}(\lambda)$ at 10 phases with, say, 10 resolution elements in the line, we still only have 100 data with which to determine a map. With such a data set, we could define, say, abundance values of a chemical element at 100 representative points on the stellar surface. This is equivalent to a resolution of about $20^{\circ}$; on a globe of the earth, such low resolution would reveal little more that the general shapes of the continents. Since different spectral lines of the same element carry almost the same information, observing several lines does not help very much. A better map can only be obtained with better spectral and phase resolution. And of course for a good map of the vector field structure over the stellar surface, we need high resolution observations of all the Stokes components. Thus to obtain really high resolution field or even abundance maps of magnetic Ap stars, quite extraordinary data sets are required. Such data sets are only now beginning to become available.

In fact, for most known magnetic Ap stars, the only available magnetic observations are of $B_{l}$. This has for many years been the preferred method of measuring the field, because (1) circular polarization in spectral lines, of opposite sign in the two line wings (or more generally, of zero value integrated in wavelength across the line) is produced only by a magnetic field, (2) this 
polarization provides the most sensitive means of detecting the modest but structurally simple fields of most Ap stars, and (3) no detailed knowledge of the intrinsic line profile is needed to derive the field moment of interest. Thus for most $\mathrm{Ap}$ and $\mathrm{Bp}$ stars, the only constraint available on the stellar magnetic geometry is the variation $B_{l}(\phi)$ with phase $\phi$.

For some stars, however, considerably more information is available. For a few stars, detailed spectral observations of Stokes $\bar{I}(\lambda)$, together with measurements of the longitudinal field, have been used to construct rough models of magnetic geometry (Landstreet 1988; Landstreet et al. 1989). Glagolevskii et al. (1985) pioneered the use of resolved circular polarization profiles for field mapping, and Mathys is currently obtaining and analyzing high-resolution spectroscopy and circular spectropolarimetry of a number of interesting stars using the ESO CASPEC spectrograph with its Zeeman analyser (Mathys 1991, 1993, and other work in preparation). Systematic meaurements of $B_{s}$ are also underway for a fairly large sample of stars (Mathys 1990). And for the first time, significant data on the transverse Zeeman effect is being obtained (Leroy et al. 1993; Mathys 1993).

\section{SIMPLE MODELS AND INFERENCES}

We first consider some relatively straight-forward deductions and inferences that may be extracted from the data without elaborate modelling.

The observed values of $B_{l}$ for most magnetic $A p$ and Bp stars vary. With enough observing effort, these variations are invariably found to be periodic with a unique period $P$, which is the same as the period of photometric variation and of spectral variations, if such variations are observed. The observed periods cover an enormous range, from about $0.5 \mathrm{~d}$ to well over $10^{3}$ d (Catalano and Renson 1984, 1988), although the great majority of periods lie between 1 and $20 \mathrm{~d}$. The observed periods are inversely correlated with $v \sin i$. The large range of periods observed, and especially the fact that periods of over $10^{3} \mathrm{~d}$ are found in these main sequence stars, are not consistent with any known pulsation mechanism. The range of periods observed, the fact that a single period characterizes all variations, and particularly the relationship between $P$ and $v \sin i$, all strongly indicate that the observed variations of magnetic $A p$ and $B$ p stars are due to rotation with period $P$. The values of $v \sin i$ and $P$ are always consistent with stellar radii of roughly $3 R_{\odot}$ (Stepién 1989).

The variations of $B_{l}$, and of other indicators of field strength, repeat accurately with the period of rotation. There is no strong evidence for gradual change of magnetic configuration over time scales so far observed. We therefore believe that the field structure we observe is essentially frozen in the star, at least for time scales of less than about $30 \mathrm{yr}$. This fact is a great convenience for efforts to map stellar field structure, as it means that observations may be collected even over several observing seasons and subsequently phased together.

The observed variations $B_{l}(\phi)$ are sinusoidal for the majority of known magnetic Ap stars, although this is certainly not always the case. (Examples of both sinusoidal and non-sinusoidal variation of $B_{l}$ are shown by Mathys 1993, Figs. 3 and 4.) The rms field strength $\left\langle B_{l}^{2}>^{1 / 2}\right.$ is typically a few hundred $\mathrm{G}$, although a small fraction of magnetic Ap stars have fields of some $\mathrm{kG}$. The 
observed sinusoidal variation of $B_{l}(\phi)$ is consistent with the variation expected for a dipolar magnetic field structure centred in the star but with its axis of symmetry inclined at an angle $\beta$ to the rotation axis, as was demonstrated long ago by Stibbs (1950). However, measurements of $B_{l}$ are relatively insensitive to more complex field structure, such as might be described by higher multipoles (Schwarzschild 1950), and so a sinusoidal variation merely indicates that the field structure of the star has an important dipole component, not that the field is strictly dipolar.

A few stars show strongly non-sinusoidal variation of $B_{l}(\phi)(e . g$. Thompson and Landstreet 1985; Mathys 1993). Such stars may have important dipolar structure to their magnetic fields, but the fields must have a more complex structure that is at least as important (Landstreet 1990). This result hints at the possibility that a non-dipolar component to field structure may be present, perhaps at a lower level, in many magnetic Ap stars.

It is clearly of interest to fit dipole models to the stars for which $B_{l}(\phi)$ varies sinusoidally, to obtain at least a rough idea of the possible field strength and structure. Unfortunately, a sinusoidally varying $B_{l}$ provides only two numbers with which to constrain such a model, say the extreme field values $B_{l}^{+}$ and $B_{l}^{-}$. These two data are not sufficient to fix the three parameters needed to specify a simple dipole, for which on must give the inclination of the rotation axis $i$, the obliquity of the field axis $\beta$, and (say) the polar field strength $B_{d}$. Thus even a simple dipole model cannot be specified unless one has further constraints, for example a constraint on $i$ obtained from knowledge of $v \sin i$, $P$, and information about the stellar radius.

The value of $B_{l}$ is observed for about $2 / 3$ of known magnetic Ap stars to reverse sign. This turns out to provide some constraint on the distribution of $\beta$ values over the sample of stars, though not on the $\beta$ bvalue of any particular star. This is easily understood: if $\beta$ were usually nearly $90^{\circ}$, for almost any magnetic star, regardless of the inclination $i$, both magnetic hemispheres would be visible during the rotation, and the observed field $B_{l}$ would reverse sign. On the other hand, if $\beta$ were normally nearly $0^{\circ}$, for most stars the field strength would hardly be obseved to change at all. The fact that some stars show nearly constant fields, while most vary and the majority reverse sign indicates that $\beta$ is typically but not always large. Most magnetic Ap stars have strongly oblique field axes, but a few are nearly aligned (Preston 1967; Borra and Landstreet 1980).

More constraints on possible field structure may be obtained for stars for which more elaborate field measurements are available. One possibility is furnished by the small sample of stars with large fields and small projected rotation velocities, for which the mean field modulus $B$, may be measured. Measurements through a rotation cycle are available for only a few stars (most of the available data and their implications are reviewed by Landstreet 1980 ), but a major programme to obtain further data has been initiated by Mathys (Mathys 1990; Mathys and Lanz 1991). Examples of data from which measurements of $B_{s}$ may be obtained are shown by Mathys (1993). In general, the observed values of $B_{s}$ are of the order of 3 or 4 times larger than the extrema of $B_{l}$, although for a few stars the ratio is much larger. For stars with this ratio of $B_{l}+/ B_{s}$ or $B_{l}^{-} / B_{s}$, it seems clear that the dipole field component is important; a field structure dominated by a more complex structure must have a small ratio of $B_{l}$ to $B_{s}$. In addition, the fact that in some stars cleanly 
resolved Zeeman doublets with no central absorption are found (examine the profile of $\lambda 6149 \AA$ for HD 116114 in Mathys et al. 1993) clearly shows that the field structure of at least these large-field Ap stars is not composed (like the field of the sun) of a combination of field free regions and regions with strong flux, and in fact the sharpness of the two doublet components shows that the dispersion of the field modulus $B$ over the visible stellar hemisphere is not very large.

A vailablility of data giving the variation of $B_{s}$ with phase greatly improves the modelling situation. Even if $B_{s}(\phi)$ varies sinusoidally, which is often the situation, we have two more data, say the extrema of the variation, to add to the two data from the variation of $B_{l}$. In this case, we can not only define the parameters of the dipole field component, but even specify the strength of a quadrupole component, if it is assumed to be colinear with the dipole. When this is done, one invariably finds that non-zero values of both dipole and quadrupole field are required to fit the observations, and the polar strength of the quadrupole is typically at least about $30 \%$ of the polar field of the dipole. The most obvious way in which the field structure differs from purely dipolar is clearly visible in stars for which the line of sight passes near both poles, so that $B_{l}$ reverses more or less symetrically. In such stars, the value of $B_{s}$ observed at the two poles is typically rather different, although for a pure dipole, $B_{s}$ should be the same at both poles. That is, we find a field structure in which the local values of $B$ are rather larger at one pole than at the other.

To get further constraints on the field structure, observations sensitive to other moments of the field are required. Two very promissing approaches have begun to be exploited recently. Mathys, as described in this volume (Mathys 1993), is using a method of line profile moments with spectrophotometric and spectropolarimetric data to extract several field moments, which will ultimately be used to model field structure in a large sample of stars. With these data, he already can show the general inadequacy of a simple dipole field structure to account for the majority of the stars he has observed. A second very interesting approach is being taken by Leroy (Leroy et al. 1993; see also Landolf et al. 1993), who has been observing a number of stars for variable broadband linear polarization produced by the transverse Zeeman effect in saturated spectral lines. An observable effect has been discovered in several stars; the effect is detectable essentially only in rather cool Ap stars $\left(T_{e} \leqslant 9000\right.$ $\mathrm{K})$ with strong $\left(B_{l} \geq 1 \mathrm{kG}\right)$ fields. This programme represents the first time the transverse Zeeman effect has been observed in a significant sample of stars. Such data are expected to be quite valuable for modelling, as the direction and strength of the linear polarization are approximately proportional to the projection of the mean transverse field on the sky, so that strong constraints on the angles $i$ and $\beta$ may be obtained, as well as direct information about the strength of the transverse field component.

As previously noted, many magnetic Ap stars are also spectrum and/or light variables. These variations clearly indicate the occurence over the stellar surface of important variations in chemical abundance which are frozen into the magnetic field structure. The observed spectrum variations are directly produced as regions of different chemical abundances appear on the visible hemisphere. It is believed that the light variations are due to corresponding changes in atmospheric structure caused by variations in ultraviolet continuous 
opacity and in line blanketing, which change the wavelength distribution of the emitted flux and thus the brightness as observed in given passbands, as the star rotates.

This non-uniformity of abundances over the surface of a star clearly leads to extra problems in the determination of field structure. If the field is measured using lines of an element which is not uniformly distributed over the stellar surface, the inferred field moment will not be smoothly weighted over the visible hemisphere, and in fact it is necessary to know - or determine - the distribution of the element(s) whose line are used. One direct way to evade this problem is to measure the field strength using lines of $\mathrm{H}$, the one element expected to be essentially uniform in distribution over the surface simply because of its numerical preponderance over all other elements. The Balmerline Zeeman analyser technique (Borra and Landstreet 1980; Landstreet 1992) was developed partly to take advantage of this simplicity. However, interpretation of Balmer line measurements is not as straight-forward as was originally expected, because the simultaneous occurrence of Zeeman and Stark broadening makes it very difficult to calculate the precise response of the hydrogen atom to an applied magnetic field (Mathys 1989).

Some simple conclusions may be drawn without detailed modelling of abundance distributions. Even stars in which lines of some elements are extremely variable also usually show other elements whose lines are relatively constant. It appears that usually at least some elements are distributed more or less uniformly over the stellar surface. In some stars (e.g. HD 32633 or HD $137909=\beta \mathrm{CrB}$ ), none of the spectral lines vary much, and all elements seem to be fairly uniformly distributed. For stars which are spectrum variables, light and spectrum variations are normally phased in the sense that extrema of both tend to coincide (as one would expect if the light variations are due to the non-homogeneous distribution of some elements). Furthermore, with a few exceptions, the extrema of light and/or spectrum variations coincide closely with extrema of the variation of $B_{l}$. It seems quite plausible that in such cases, the distribution of elements is roughly axisymmetric around the field axis, with patches of over- or under-abundance near the magnetic poles.

\section{DETAILED MODELLING}

Large-field stars for which more extensive data than merely the variation of $B_{l}(\phi)$ are available (such as $B_{s}(\phi)$, detailed line profiles observed through the rotation cycle, and/or spectropolarimetry) may be modelled more accurately, but this is not a simple task. In general, it is essential to establish that the element whose spectral lines are used to study the field is distributed fairly uniformly over the visible stellar surface, or to solve for the distribution of the element as part of the computation of determining the field structure. In some cases it appears that significant depth variations of abundance occur as well (Babel 1992; Babel and Lanz 1992), and this may have to be taken into accout to obtain correct line profiles.

If the available data concerning the field is essentially moments of the field distribution $\left(B_{l}, B_{s}\right.$, or the moments that Mathys derives from his spectropolarimetry - see Mathys 1993), the most straight-forward procedure for field modelling is probably to assume a parameterized model such as 
an expansion in colinear multipoles. If enough information is available to constrain a more complex field structure and the symmetry of the obvious simple models does not seem to be present, one may work with other types of parameterized field distributions such as the generalized offset dipoles of Stift (1975). The parameters of the expansion are then varied to obtain agreement with the observed field moments as a function of rotational phase.

This same strategy may be used for comparison with high-resolution spectroscopy or spectropolarimetry. If the model has more degrees of freedom than are defined by the data, then a further condition (e.g. using the smallest number of multipoles possible, or the maximum entropy condition) must be invoked to chose the preferred model. Strategies and uniqueness are discussed by Stift (1991). In making comparison with observed spectra, it is of course necessary to calculate the expected line profiles in the presence of relatively strong magnetic fields. This problem has been discussed from a practical point of view (in LTE) by Unno (1956), Wittmann (1974, 1977), and Landi Degl'Innocenti (1976). Calculations of line profiles of magnetic Ap stars have been carried out by Freedman (1978) for $\beta$ CrB, by Stift (1985) for the purpose of examining the diagnostic content of the profiles, and by Landstreet (1988), Landstreet et al. (1989), and Bohlender (1989) for modelling.

Field structure determination based on a multipole expansion fitted to measurements of the mean longitudinal field and of suitable magnetically sensitive line profiles in a series of spectra have been reported for the Ap SrCrEu star HD $65339=53 \mathrm{Cam}$, which has a mean field modulus which varies between 10 and $17 \mathrm{kG}$ (Landstreet 1988), and of the hotter Bp Si star HD 215441 = Babcock's star, which has a $B_{s}$ of about $35 \mathrm{kG}$ (Landstreet et al. 1989). Modelling of these two stars was carried out by assuming a field structre described by a superposition of colinear dipole, quadrupole, and octupole fields, with the adjustable parameters the polar field strengths $B_{d}, B_{q}$, and $B_{o}$, and the two angles $i$ and $\beta$. The parameters were adjusted to fit the observed variation of $B_{l}$. In the case of $53 \mathrm{Cam}$, they were simultaneously fitted to the profiles of one magnetically insensitive line and two strongly magnetically broadened lines of $\mathrm{Cr}$, an element which is observed to vary very little in the spectrum of this star. The variation of the $\mathrm{Cr}$ abundance with magnetic colatitude was determined (with very low resolution) at the same time. For HD 215441, the field structure was chosen to fit a completely resolved line of Si III with a simple triplet structure; again the distribution of the element was determined simultaneously with the field structure, assuming axisymmetry around the magnetic axis. Then for both stars, with the best fit field structure fixed, the distribution in magnetic colatitude of several other elements could be determined.

The best-fitting field structures found for these two stars do not give perfect fits to the observed line profiles, and so must be regarded as at best reasonable approximations to the real field distributions. However, some interesting conclusions can be drawn from the modelling. First, the dipole component is the dominant one, but higher multipoles are quite important. In fact, a substantially better fit is obtained in both cases with three components than may be found neglecting the octupole. For both stars it appears that the local field strength is quite different at the two magnetic poles. For these two stars, there is no significant evidence of departure from axi-symmetry (this is not true for other stars, e. g. Mathys 1993). It is clear for 53 Cam that at 
least some of the line profile variations observed are due to changes in the field strength; however, real variations in local abundance of the order of two dex occur for the elements $\mathrm{Ca}$ and $\mathrm{Ti}$, while other elements $(\mathrm{Cr}, \mathrm{Fe}, \mathrm{Sr}$ ) show much smaller variations. For HD 215441, it appears that all elements vary in local abundance by roughly one dex just over the one hemisphere of the star that is observed. In $53 \mathrm{Cam}$, the angles $i$ and $\beta$ found are both large, about $65^{\circ}$ and $80^{\circ}$. For HD 215441 , these angles are considerably smaller, both near $35^{\circ}$. Further modelling of $\mathrm{Ca}$ in $53 \mathrm{Cam}$, assuming the vertical stratification predicted by a model of diffusion in the presence of a wind, has been carried out by Babel (1992). As he discusses in his paper at this meeting (Babel 1993), it appears that the results of this modelling furnish evidence for vertical stratification and indirectly for a weak stellar wind in the atmosphere of this star (see also Babel and Lanz 1992).

Some results using this same method have also been reported for the magnetic He-strong stars HD $36485=\delta$ Ori C and HD 58260. The longitudinal field of each of these stars is approximately constant, so either $i$ or $\beta$ is quite small, and we see the magnetic field from a nearly constant magnetic colatitude as the star turns, rather than looking at it from near one pole to near the other. As a result, the field structures are only moderately well constrained (Bohlender 1989).

It is clearly desirable to map field structures without assuming a simple model of those structures which may not be appropriate. The fields of real stars may not actually be axisymmetric, and they may be poorly represented by a low-order multipole expansion. This is an area of mapping in which rapid progress may be expected in the near future. Of course, as emphasized above, for a detailed assumption-free map to be meaningful, it is essential that it be based on an extremely rich data set with both high spectral and phase resolution, and circular and linear polarimetry.

Most of the mapping work done to date on magnetic Ap stars without assuming a model geometry has been mapping of abundance distributions on the surfaces of stars with intrinsically weak magnetic fields, using the technique of Doppler imaging. In this method, the surface of the star is divided into a large number of small regions, and the abundance of the element under study is determined for each (visible) region from the constraints provided by the variation with rotational phase of one or several line profiles. A particular profile will vary as regions of high or low abundance move onto the visible hemisphere because of stellar rotation. This variation effectively provides longitude information about the location of patches or spots. If the inclination $i$ is substantially less than $90^{\circ}$, the fraction of the rotation period that a spot is visible also provides some information about its latitude. In addition, if the star is rotating rapidly enough that Doppler broadening is important, abundances patches located near the rotational equator will have significant changes in radial velocity (from the blue wing of the line as the patch comes into view, to the red wing as it disappears). In contrast, a patch near one of the rotational poles will have little radial velocity variation. Thus, the radial velocity variations furnish additional clues to the latitude of observed spots. Mapping based on solving for the abundance values at many representative surface points by fitting observed line profiles has developed greatly in the past few years. Good reviews are those of Khokhlova (1985) and Vogt (1988). Recent work in this field is discussed by Hatzes (1993), Khokhlova (1993), and 
Wehlau and Rice (1993). Remarkably detailed maps can now be obtained for the distributions of several elements over the surfaces of the stars most suitable for mapping.

An important limitation of most of mapping of abundance distributions by Doppler imaging done to date is that it has focussed on magnetic stars with weak fields. Mapping of abundance distributions is much simpler for weak field stars than for strong field ones. This is because in the weak field limit, the local line profiles are not significantly affected by the magnetic field (see the discussion of the low-field solution to the equations of transfer in the second section of this paper), and may be represented by a simple, oneparameter form. With a strong field, the local line profile and strength depends not only on the local abundance but also on the local (vector) field, which must therefore be determined at the same time the abundance map is being found. Furthermore, the local profile must be obtained by solving the full set of equations of transfer rather than just the single non-magnetic equation. An additional complication produced by a strong field is that it widens the local line profile significantly, thus mixing up the information contained in the Doppler displacements of contributions to the integrated profile from various parts of the disk. To avoid these complications, most of the work done to date on Doppler imaging has concentrated on weak-field stars. But as I have emphasized in the second section of this paper, in this case the information available to constrain the field is very limited indeed, and it is generally not possible to determine a well-defined field map for comparison with the derived abundance maps.

This limitation of the usual Doppler imaging is now being addressed. Several years ago, Glagolevskii et al. (1985) showed that resolved circular polarimetry of spectral lines could be used to map the field structure of $\alpha^{2}$ $\mathrm{CVn}$, and Piskunov is developing a more sophisticated Doppler imaging code for field determination. A similar field mapping code has been developed by Donati and several collaborators. So far, this code has mainly been applied to cool stars. A splendid example of the new possibilities for determination of magnetic geometry is shown by the field map derived for the cool spotted K1 IV star in the RS CVn binary system HR 1099 by Donati et al (1992), on the basis of the rotational modulation of $\bar{I}$ and $\bar{V}$ line profiles observed with high resolution. It is clear that within a few years, we will see a major improvement in the quality of the available field maps. These will undoubtedly form an important basis of discussion at the next meeting on Ap and Bp stars.

\section{REFERENCES}

Adelman, S. J. 1993, in Peculiar versus Normal Phenomena in A-type and Related Stars, ed. F. Castelli and M. M. Dworetsky, Publ. A. S. P. Conference Series (this volume).

Babel, J. 1992, Astr. Ap., 258, 449.

Babel, J. 1993, in Peculiar versus Normal Phenomena in A-type and Related Stars, ed. F. Castelli and M. M. Dworetsky, Publ. A. S. P. Conference Series (this volume).

Babel, J. and Lanz, T. 1992, Astr. Ap., in press.

Bohlender, D. A. 1989, Ap. J., 346, 459. 
Borra, E. F. and Landstreet, J. D. 1977, Ap. J., 212, 141.

Borra, E. F. and Landstreet, J. D. 1978, Ap. J., 222, 226.

Borra, E. F. and Landstreet, J. D. 1980, Ap. J. Suppl., 42, 421.

Catalano, F. A. and Renson, P. 1984, Astr. Ap. Suppl., 55, 371.

Catalano, F. A. and Renson, P. 1988, Astr. Ap. Suppl., 72, 1.

Cowley, C. R. 1991, Evolution of Stars: the Photospheric Abundance

Connection, ed. G. Michaud and A. Tutukov, Kluwer Academic

Publishers, p. 183.

Cowley, C. R. 1993, in Peculiar versus Normal Phenomena in A-type and Related Stars, ed. F. Castelli and M. M. Dworetsky, Publ. A. S. P. Conference Series (this volume).

Donati, J.-F., Brown, S. F., Semel, M., Rees, D. E., Dempsey, R. C., Matthews, J. M., Henry, G. W. and Hall, D. S. 1992, Astr. Ap., in press.

Dworetsky, M. M. 1993, in Peculiar versus Normal Phenomena in A-type and Related Stars, ed. F. Castelli and M. M. Dworetsky, Publ. A. S. P. Conference Series (this volume).

Freedman, R. S. 1978, Ap. J., 224, 910.

Glagolevskii, Yu. V., Piskunov, N. E. and Khokhlova, V. L. 1985, Sov. Astr. Lett., 11(3), 154.

Hatzes, A. P. 1993, in Peculiar versus Normal Phenomena in A-type and Related Stars, ed. F. Castelli and M. M. Dworetsky, Publ. A. S. P. Conference Series (this volume).

Khokhlova, V. L. 1985, Sov. Sci. Rev. E, Ap. Sp. Phys., 4, 99.

Khokhlova, V. L. 1993, in Peculiar versus Normal Phenomena in A-type and

Related Stars, ed. F. Castelli and M. M. Dworetsky, Publ. A. S. P.

Conference Series (this volume).

Landi Degl'Innocenti, E. 1976, Astr. Ap. Suppl., 25, 379.

Landolfi, M., Landi Degl'Innocenti, E., Landi Degl'Innocenti, M. and Leroy, J.-L. 1993, in Peculiar versus Normal Phenomena in A-type and Related Stars, ed. F. Castelli and M. M. Dworetsky, Publ. A. S. P. Conference Series (this volume).

Landstreet, J. D. 1980, Astr. J., 85, 611.

Landstreet, J. D. 1988, Ap. J., 326, 967.

Landstreet, J. D. 1990, Ap. J. (Letters), 352, L5.

Landstreet, J. D. 1992a, in The Atmospheres of Early-type Stars, ed. U. Heber and C. S. Jeffery, Springer-Verlag, p. 170.

Landstreet, J. D. 1992b, Astr. Ap. Rev., in press.

Landstreet, J. D., Barker, P. K., Bohlender, D. A. and Jewison, M. S. 1989, Ap. J., 344, 876.

Leroy, J.-L., Landstreet, J. D., Landi Degl'Innocenti, E. and Landolf, M. 1993, in Peculiar versus Normal Phenomena in A-type and Related Stars, ed.

F. Castelli and M. M. Dworetsky, Publ. A. S. P. Conference Series (this volume).

Mathys, G. 1989, Fund. Cos. Phys., 13, 143.

Mathys, G. 1990, Astr. Ap., 232, 151.

Mathys, G. 1991, Astr. Ap. Suppl., 89, 121.

Mathys, G. 1993, in Peculiar versus Normal Phenomena in A-type and Related Stars, ed. F. Castelli and M. M. Dworetsky, Publ. A. S. P. Conference Series (this volume).

Mathys, G. and Lanz, T. 1992, Astr. Ap., 256, 169. 
Mathys, G., Landstreet, J. D. and Lanz, T. 1993, in Peculiar versus Normal Phenomena in A-type and Related Stars, ed. F. Castelli and M. M.

Dworetsky, Publ. A. S. P. Conference Series (this volume).

Preston, G. W. 1967, Ap. J., 150, 547.

Preston, G. W. 1970, Ap. J., 160, 1059.

Rees, D. E. 1987, in Numerical Radiative Transfer, ed. W. Kalkofen, Cambridge University Press, p. 312.

Ryabchikova, T. A. 1991, Evolution of Stars: the Photospheric Abundance Connection, ed. G. Michaud and A. Tutukov, Kluwer Academic Publishers, p. 149.

Schwarzschild, M. 1950, Ap. J., 112, 222.

Stepién, K. 1989, Astr. Ap., 220, 105.

Stibbs, D. W. N. $1950, M$. N. R. A. S., 110, 395.

Stift, M. J. $1975, M . N$. R. A. S., 172, 133.

Stift, M. J. 1985, M. N. R. A. S., 217, 55.

Stift. M. J. 1991, Astr. Ap., 251, 139.

Takada-Hidai, M. 1991, Evolution of Stars: the Photospheric Abundance

Connection, ed. G. Michaud and A. Tutukov, Kluwer Academic Publishers, p. 137.

Thompson, I. B. and Landstreet, J. D. 1985, Ap. J. (Letters), 289, L9.

Unno, W. 1956, Publ. Astr. Soc. Japan, 8, 108.

Vogt, S. S. 1988, in The Impact of Very High S/N Spectroscopy on Stellar Physics, ed. G. Cayrel de Strobel and M. Spite, Kluwer Academic Publishers, p. 253.

Wehlau, W. H. and Rice, J. B. 1993, in Peculiar versus Normal Phenomena in A-type and Related Stars, ed. F. Castelli and M. M. Dworetsky, Publ. A. S. P. Conference Series (this volume).

Wittmann, A. 1974, Solar Phys., 35, 11.

Wittmann, A. 1977, Astr. Ap., 54, 175.

Wolff, S. C. and Wolff, R. J. 1970, Ap. J., 160, 1049. 